\title{
“LA CAUSA DELLE DONNE" Y LOS DERECHOS POLÍTICOS DE LAS MUJERES DURANTE EL TRIENIO JACOBINO ITALIANO
}

\author{
“LA CAUSA DELLE DONNE” AND POLITICAL RIGHTS OF WOMEN IN THE \\ JACOBIAN ITALIAN TRIENNUM
}

Milagro Martín Clavijo

Universidad de Salamanca

\begin{abstract}
ReSumen:
Durante el Trienio jacobino, 1796-1799, en Italia se empiezan a implantar los principios fundamentales para el ser humano que se encuentranenlabasedela Revoluciónfrancesa. En estos años un número considerable de mujeres va a tomar parte activa en reuniones públicas de carácter político, social y cultural y se convierten en protagonistas de discursos importantes, que suscitarán, entre algunos, la admiración y, entre otros, el escándalo. Entre los discursos más importantes se encuentra el de La causa delle donne, probablemente de Annetta Vadori: una reivindicación explícita y determinada, sin tapujos ni eufemismos, sin medias palabras, a la igualdad entre sexos.
\end{abstract}

\section{Palabras claves:}

Trienio jacobino, Annetta Vadori, patriotas, derechos políticos de las mujeres.

\section{Abstract:}

During the Jacobin Triennium, 17961799, in Italy starts the implementation of fundamental human principles at the base of the French Revolution. In these years a large number of women actively participate in political, social and cultural meetings and make public speeches, which would create, among some, admiration and, among others, scandal. One of the most important speeches is La causa delle donne, probably written by Annetta Vadori: an explicit and specific claim, without euphemisms, without mincing words, of equality between the sexes.

\section{KEY WORD:}

Jacobin Triennium, Annetta Vadori, patriots, political rights of women. 


\section{El Trienio Jacobino ItALIANO}

1796-1799 son los años que en Italia reciben el nombre de Trienio patriótico, jacobino o revolucionario. Poco tiempo, es verdad, pero se trata de años fundamentales para la puesta en marcha de la implantación de principios fundamentales para el ser humano, los que se encuentran en la base de la Revolución francesa.

Durante este trienio algunas repúblicas italianas -la República Cisalpina, la Véneta, la Ligur, la Romana, la Napolitana- instauran en Italia instituciones similares a las de la Francia revolucionaria. De esta manera, se convierten en territorios satélites de ésta o directamente bajo su influencia. Es ahora que comenzará un importante debate sobre la independencia nacional, sobre la educación, sobre las instituciones políticas, las minorías excluidas de la vida pública y los derechos de los ciudadanos. Este debate va acompañado de importantes propuestas de reformas que, pese a su breve duración van a dejar su huella y serán la base de proyectos futuros.

En este propósito común de renovación de la vida en general -tanto de mejora económica, como de mayor libertad e influencia política- participan las mujeres como hasta ahora no se había hecho nunca en Italia. Ellas también abogan por la revolución que significa también, o sobre todo,

\section{l'abbattimento di leggi e consuetudini familiari che le discriminavano doppiamente, come figlie e spose, che le volevano mogli o monache forzate in nome della salvaguardia dei patrimoni familiari, che le vedevano escluse dall'asse patrimoniale in base ai principi del maggiorascato, che le privavano dell'istruzione, che limitavano drasticamente le loro scelte personali. [...] Molte donne insomma vedevano nella Rivoluzione l'inizio di un processo di cambiamento sociale che, nel cancellare le diseguaglianze, andava a ridisegnare anche una nuova condizione femminile (Filippini, 2006: 89).}

\section{El aprendizaje político de las mujeres en territorio véneto}

Es en este clima de profundo optimismo en la posibilidad de hacer una revolución en todos los campos de la vida del individuo que tenemos que enmarcar uno de los textos más importantes sobre los derechos políticos de las mujeres, La causa della donna, escrito por una mujer en la Venecia del periodo de la Municipalidad y que abarca un breve periodo, desde el 12 de mayo de 1797 al tratado de Campoformio, 17 octubre 1797. Durante estos meses se constituye en Venecia, como en otras ciudades vénetas, un gobierno que sigue el modelo francés y en el que entrarán exponentes de distintas clases sociales.

Las ideas jacobinas ya se habían difundido en territorio véneto desde principios de la década de 1790, especialmente entre los ilustrados de la burguesía y de la aristocracia, pero también entre periodistas, científicos y artesanos que se reunían en cafés, casinos, salones, talleres y tiendas. En estas reuniones participan también muchas mujeres escritoras, periodistas, artistas, cultas e ilustradas, entre las que podemos destacar Elisabetta Caminer Turra en Vicenza, Francesca Maria Bragnis Capodilista y Arpalice Papafava en Padua; Maria Querini Benzon, Isabella Teotochi Albrizzi, Giustina Renier Michiel y Anna Vadori en Venecia.

Algunas de estas mujeres van más allá de los salones o reuniones informales para entrar de lleno en las nuevas instituciones democráticas y asumir en ellas un papel político visible. Su participación en las Sociedades de Instrucción Pública y en las Sociedades Patrióticas va a ser fundamental para el aprendizaje político de las mujeres. Es allí donde se van a difundir los nuevos principios democráticos y desde donde se intenta educar al pueblo en las nuevas ideas. Estas sedes sirven también de intermediarias con el gobierno de las municipalidades $\stackrel{1}{\text {. }}$

Estas instituciones, que surgen en todas las ciudades del Véneto, tienen una actividad muy intensa de debate tanto político y social, como cultural. En la municipalidad de Venecia un papel fundamental lo tendrán Annetta Vadori, Cecilia Tron y Gioseffa Cornoldi Caminer, pero también otras mujeres de las que sólo conocemos sus iniciales o que son anónimas, pero que nos han dejado importantes textos para la querella de las mujeres: I.P.M. (1797), y la anónima de Istruzioni d'una libera cittadina alle sue concittadine (1797).

Se trata de una presencia innovadora de mujeres que toman parte activa en las reuniones y que protagonizan discursos públicos, que suscitarán, entre algunos, la admiración y, entre otros, el escándalo. Entre los discursos más importantes en territorio véneto están los pronunciados en mayo de 1797 por la ciudadana Fulvia Mattei, miembro de la Società Patriottica, Dell'educazione che si deve dare alle donne, y el de La causa delle donne, probablemente de Annetta Vadori.

Estas mujeres van a subir a la tribuna para reclamar sus derechos, para llegar a la igualdad universal que tanto se predica, para reafirmar la capacidad de la mujer para ejercer sus derechos civiles. En estos discursos queda patente que eran conscientes de que la cuestión feminista era un aspecto más de la lucha por los derechos universales y que ése era el momento para hacerlo: antes las "ideas revolucionarias" estaban perseguidas, por lo que tenían que aprovechar esos años de cambio de sistema político para alzar la voz y reivindicar los derechos que le son naturales, como poco antes lo habían hecho las ciudadanas francesas. Pero el caso de las patriotas italianas es distinto al de sus vecinas, ya que en los años del Trienio ya es un hecho el fracaso de

1 Las municipalidades eran "enti invertiti di funzioni amministrative autarchiche di carattere locale, operanti per lo più sotto la dipendenza e il controllo delle autorità militari di occupazione, rappresentano assai frequentemente l'inizio di quel processo di democratizzazione e di creazione $\mathrm{d} i$ ordinamenti costituzionali che accompagnò l'avanzata francese" (Pisano-Veauvy, 1994: 200). 
las reivindicaciones de las francesas y muchas de ellas han sufrido la persecución, la prisión o incluso han sido ejecutadas. Por tanto, estas mujeres italianas, portavoces de ideas ciertamente feministas a finales del siglo XVIII, son conscientes de la precariedad de su situación y del riesgo que corren al afirmar sus ideas públicamente.

Los discursos de las patriotas están dirigidos al público que asiste a las reuniones de los círculos. Éste estaba formado por líderes del Jacobismo en Italia y también por simpatizantes, en su gran mayoría hombres cultos y de clases sociales altas o medias. Pero también asistían mujeres, la mayoría con cultura, pero algunas también eran mujeres del pueblo, analfabetas, como se señala en algunos de estos discursos 2 .

Una parte considerable de estas lecturas públicas están dirigidas exclusivamente a las mujeres, de forma clara e insistente. Otro grupo de textos tienen como destinatarios a los ciudadanos, en masculino plural. A veces, se hace explícito que se trata de hombres, otras veces se deja el término en la ambigüedad. Hay que destacar en este sentido que los dos textos más reivindicativos del trienio -el de Carolina Lattanzi (1797) y La causa delle donne (1797)- están dirigidos a un público fundamentalmente masculino.

Como vemos, en estos círculos se toleraba, e incluso se aceptaba de buena gana, la presencia de las mujeres y el hecho de que tomaran la palabra directamente. De hecho si algunos de estos discursos se publicaron es porque el público presente los consideró dignos. Por otro lado, normalmente lo que se decía en ellos no suponía un peligro real para los patriotas: servía para ayudar a incrementar la participación femenina en la lucha por la república, pero sin atentar explícitamente contra sus privilegios como hombres. Es verdad que la participación de las mujeres en la vida política a través de los círculos no fue del agrado de todos, pero algunos patriotas van a mostrarse favorables y van a fomentar su participación activa en ellos.

\section{La causa delle donne y los problemas de autoría}

Algunos estudiosos han atribuido La causa delle donne, 1797, a una autora véneta y con mucha probabilidad a Annetta Vadori ${ }^{3}$. De su vida tenemos poca documentación. Sabemos que nació en Venecia en 1761, en el seno de una familia burguesa y que desde joven se acerca a las ideas de la Ilustración y de la Revolución. Participa en el círculo jacobino del farmacéutico Vincenzo Dandolo (Bustico, 1917: 370-78) y, por

2 Cfr. los discursos de Claudia Stella (1986:1103-1105) y de Teresa Negri (1986: 1095-1101) en la comida patriótica que tuvo lugar en Boloña en 1798.

3 Sobre la atribución de la autoría a Anna Vadori cfr. Dumas (1964: 464). En Pillinini (1990: 92) se identifica La causa delle donne como el discurso que Anna Vadori pronunció en la Società di pubblica istruzione. A. Buttafuoco (1991: 99-106) la señala como posible autora, a pesar de que no cree que se trate de un texto de la zona véneta. Apoyan también esta atribución Plant (2002: 29), Romanelli (1997) y Filippini (2006: 101), entre otros. Emanuele Antonio Cicogna (1847) atribuye a Anna Vador también la autoría de algunos escritos anónimos de este periodo. ello, la vigilará la policía desde 1792. Es amiga de Elisabetta Caminer, Alberto Fortis y Tommaso Gallino.

A los 25 años se casa con Mattia Butturini, comediógrafo del teatro de San Benedetto. Con él colabora y escribe textos y poesías (Filippini, 2006: 86-87).

Tras la proclamación de la Municipalità Provvisoria, asume un papel importante en la Società di Pubblica Istruzione junto a otras mujeres como Cecilia Tron, Gioseffa Cornoldi Caminer y Fulvia Mattei. En esta sede se bate por los derechos de las mujeres. En 1797 sube a la tribuna después de haber jurado "viver libera o morire". Se trata de un gesto claramente simbólico en el que los ciudadanos juraban que defenderían la patria con su vida, pero dice mucho el hecho de que se tratara de una mujer, ya que tal juramento implicaba la equiparación de la mujer al hombre. De hecho, tras la promesa Vadori reivindica la igualdad de derechos para todas las mujeres. La asamblea la aclama y decide que se publique su discurso. Su intervención y su posición anima un fuerte debate en la Società. En este mismo año también lleva a cabo la traducción de la obra de Aubert du Bayet, Rapporto d'una festa civica celebrata in Costantinpoli, que lee también en una sesión de la asamblea (Filippini, 2006: 99).

Después del tratado de Campoformio, 17 octubre 1797, con el que Napoleón cede Venezia y el territorio véneto a Austria, concluye esta estación reformista. En enero entra la armada austro-rusa en las ciudades vénetas. Los jacobinos son perseguidos, capturados, asesinados o se dan a la fuga o al exilio. Annetta deja la ciudad e inicia una vida de viajes e incertidumbres ${ }^{4}$.

Como muchos otros jacobinos, en un primer momento se dirige a Lombardia, concretamente a Pavía, con el marido, que enseña lengua y literatura griega. Se sabe que en 1805 se divorcia y que poco después se casa con el médico Giovanni Rasori, importante teórico del jacobismo científico. Su matrimonio no durará mucho. Más tarde la encontramos en Milán, junto a Tommaso Gallino, abogado y miembro del Consiglio legislativo de la República Italiana.

En esta ciudad lombarda en 1809 abre su salón a intelectuales y literatos de su época, entre los que se encuentran Cesarotti, Pindemonte, Monti y también Foscolo, autor con el que mostrará públicamente un profundo desacuerdo. En Il Salotto Della Contessa Maffei e la Società Milanese (1834-1886) se habla de las reuniones organizadas por la veneciana y se cita a Giovanni Resini que habla de ella como "una vera Aspasietta" (Barbiera, 1895: 32-34), comparándola así con Aspasía de Mileto, la compañera de Pericles.

Años más tarde, junto con los exiliados cisalpinos, se establecerá en París donde será introducida en la corte francesa por el famoso improvisador Francesco Gianni. De esta

4 Sobre su vida sucesiva cfr. Cosmacini (2002), Rao (1992) y Angelini (2007). 
manera, se la situa muy cerca de la madre de Napoleón. Sin embargo, tras la conjura antinapoleónica de Ceracchi, a pesar de que se demuestra que ella no tuvo nada que ver, es el propio Napoleón el que la obliga a dejar Francia. Vuelve a Italia, otra vez a Milán, donde vuelve a abrir su salón a los intelectuales. En estos años se produce el reencuentro con su amiga Cecilia Tron.

Sin embargo, sus condiciones económicas van a ir empeorando. En 1807 se establece en Nápoles como institutriz de los príncipes Pignatelli, pero por poco tiempo. En 1832 muere sola en la más absoluta miseria.

Annetta Vadori fue una mujer de carácter, culta, una gran oradora y ciertamente hermosa; todo esto se nos revela en los epistolares de algunos literatos de su tiempo que la recuerdan, algunos con admiración, como Monti, otros con oposición frontal, como Foscolo. Se la conoció como la "Aspasia veneziana": por su anticonformismo, su libertad personal y sexual. Son estos aspectos de su intimidad los que le van a hacer de Annetta la diana de sus críticas y los que la condenarán al más profundo silencio durante el siglo XIX y gran parte del XX.

Las cosas están cambiando en los últimos decenios: en el volumen L'altra metà del Risorgimento. Volti e voci di patriote venete se nos presenta a Annetta Vadori como las más anciana de las patriotas y se la califica como la "rivoluzionaria vagabonda" (FilippiniGazzetta, 2011, 114-116).

4 .La causa delle donne y la igualdad entre hombres y mujeres

El discurso jacobino y la puesta en práctica de los principios de la Revolución francesa llevaban directamente a la reivindicación de la igualdad entre sexos, como constatan en sus discursos y en sus escritos muchas de estas mujeres. Sin embargo, será esta temática la que evidenciará claramente cómo la cuestión femenina se dejaba por regla general al margen del debate político, por lo que las pocas mujeres que alzarán su voz para reivindicar sus derechos contarán con la fuerte oposición de gran parte de sus compañeros de ideología.

Sin embargo, en La causa delle donne nos encontramos una reivindicación explícita y determinada, sin tapujos ni eufemismos, sin medias palabras, de la igualdad entre sexos. Se trata de un discurso que tiene la forma de texto jurídico y que está dividido en dos capítulos y éstos en un total de trece puntos.

La autora veneciana comienza con una constatación: “ègiuntal'epoca desideratissima della più bella di tutte le mode, ch'è quella della libertà ed uguaglianza", pero en medio de tanta alegría, anuncia que para las mujeres no lo es tanto, ya que éstas han quedado al margen de todo, "dunque o non tenete le donne per individui del genere umano, o pensate a felicitar di cotesto una sola metà". De ahí que se reivindique precisamente la igualdad frente a la inferioridad a la que se ha sometido a la mujer durante tanto tiempo: "pretendiamo di essere considerate al par degli uomini in tutti i pubblici interessi dell'universale reforma" (La causa delle donne, 1964: 455-6).

A partir de la explicitación directa de la tesis, la autora procede con los dos artículos que componen su discurso: "Le donne per natura sono eguali, anzi superiori agli uomini" $y$ "Le donne han diritto di essere parte di tutti i pubblici interessi della presente riforma d'Italia". Se parte, por tanto, de la igualdad entre sexos, pero sin establecer en ningún momento conflicto alguno con los hombres (Pisano, 1994: 59), para proseguir con los derechos de las mujeres.

Los dos primeros puntos son introductorios y en ellos se reivindica el derecho de las mujeres a expresarse y a presentar las propias quejas -“Il vostro eroico progetto di render liberi tutti gli uomini, questo medesimo progetto sì amato, sì commedato da tutti, ci concede la libertà di parlare ed esporre francamente le nostre ragioni"-; en el segundo adelanta en qué van a consistir las "giuste querele": las mujeres están excluidas de la esfera pública en el proyecto jacobino, "dappertutto in somma risuonano gli uomini, e le femine non si sentono mai nominare che per il solo uso matrimoniale, o quasi matrimoniale, relativo agli uomini" (La causa delle donne, 1964: 455).

Por tanto, las conclusiones a las que llega la ciudadana ${ }^{* * *}$ no pueden ser más que dos: "o non tenete le donne per individui del genere umano, o pensate a felicitar di cotesto una sola metà" (La causa delle donne, 1964: 455). La veneciana es clara y va directa al nudo de la cuestión: todos los proyectos que se están llevando a cabo para la regeneración de la república fracasarán si no participan activamente las mujeres.

Por tanto: “Noi, fratelli carissimi, nel complesso del genere umano pretendiamo di non essere inferiori: in conseguenza di questa superiorità, o almeno eguaglianza, pretendiamo di essere considerate al par degli uomini in tutti i pubblici interessi dell'universale riforma. Queste sono le nostre due pretensioni, questi i due articoli della nostra causa" (La causa delle donne, 1964: 456).

Su tesis es la igualdad entre hombres y mujeres, una igualdad efectiva y no sólo teórica y abstracta. Hasta el momento se puede muy bien hablar de esclavitud femenina ${ }^{5}$, ya que han estado siempre sometidas al poder cultural y social del hombre (Pisano-Veauvy, 1994: 203). Se consideraba que la mujer era inferior a éste y, por tanto, se establecían funciones distintas para cada sexo. De esta manera, a la mujer se la recluía en la esfera privada y se la excluía sistemáticamente de la pública.

Hay que partir, por tanto, de la igualdad entre hombres y mujeres. La mayoría de los textos escritos por éstas parten de la genealogía femenina, es decir, con el ejemplo ilustrativo de un importante número de mujeres que han demostrado que están a la

5 Este será precisamente el título del discurso de Carolina Lattanzi, Schiavitù delle donne, 1797. 
altura de los hombres. La autora veneciana traza también una genealogía, aunque afirma que no pretende convencer con la historia, sino con la filosofía y la razón siguiendo "un'argomentazione fisico-giusnaturalistica di stampo rousseauviano" (Rosa, 2009: 122): ya desde la creación del mundo y del primer hombre se hace evidente que éste necesitaba "la compagnia d'un'altra creatura ragionevole, la qual fosse nel tempo stesso e differente da lui ed eguale a lui" (La causa delle donne, 1964: 457-8).

De esta manera, introduce el concepto de la igualdad en la diferencia para el hombre y la mujer: "Gli organi della generazione, ed i naturali affetti che l'accompagnano, richiedevano senza dubbio una creatura differente: ma tutte l'altre passioni ed inclinazioni dirette alla società richiedevano una creatura eguale". Con la creación de la mujer se conseguía que estuviera "maravigliosamente equilibrata nella donna e nell'uomo la differenza e l'eguaglianza: cioè la differenza de' sessi, e l'eguaglianza delle nature. Eccettuate dunque le potenze produttrici, e tuttociò che con esse ha necessaria relazione, l'uomo e la donna nel rimanente sono per natura egualissimi" (La causa delle donne, 1964: 458).

Si la mujer y el hombre son iguales por naturaleza, habrá de despojarse de todos los prejuicios y costumbres que nos han llevado durante tantos siglos a considerarlas inferiores. Por eso, exhorta a sus conciudadanos filósofos a que pesen "la nostra e la vostra costituzione intrinseca nella bilancia della filosofia, e vedrete che la nostra soggezione non solo è posteriore alla natura, ma in questo suo vero aspetto non esce mai dai confini matrimoniali, e non ha relazione alcuna cogli affari pubblici della società, che formano tutto l'oggetto delle nostre lagnanze" (La causa delle donne, 1964: $459)$.

En el punto número ocho, la ciudadana veneciana se centra en una de las tradicionales argumentaciones masculinas sobre su superioridad, la basada en la fuerza. Pero no existe sólo un tipo de fuerza, sino dos: la del cuerpo -“La ruvidezza del volto, la robustezza dei nervi, la durezza del cuore, l'ostinazione dell' intelletto, la rusticità delle membra, e per sino della voce" - y la del espíritu -“L'avvenenza del volto, la morbidezza delle carni, l'amorevolezza del cuore, l'acutezza dell'ingegno, la delicatezza di tutto il corpo, e per sino della voce- que están distribuidas con equidad entre los hombres y las mujeres" (La causa delle donne, 1964: 460). Si seguimos guiándonos por la Filosofía, no nos queda que concluir que "è più perfetta la forza dello spirito di quella del corpo" (La causa delle donne, 1964: 460)

Entonces ¿por qué todavía se considera inferior a la mujer? La clave está en la educación diferente a la que han accedido durante siglos, como expone en el punto nueve. Se reafirma, por tanto, en que no es la naturaleza la que establece la condición de inferioridad de la mujer, sino el hombre y ésta se puede superar a través de la instrucción, visto que ellas, como acaba de demostrar, están mejor dotadas para todo lo que tiene que ver con el espíritu. Los hombres, con su fuerza corporal, están allí para defender precisamente esa superioridad espiritual de las mujeres.

\section{La causa delle donne y los derechos políticos de las mujeres}

En este periodo del trienio jacobino no son frecuentes los textos centrados sobre tema de los derechos políticos de las mujeres. De hecho, pocas patriotas sienten verdaderamente la necesidad de reivindicarlos o, por lo menos, no era una de sus prioridades.

Es verdad que el concepto de ciudadanía es central en el discurso revolucionario pero, ya que se considera a la mujer fundamentalmente como madre, lleva como consecuencia la dificultad de que ésta se convierta en una ciudadana activa. Por tanto, lo habitual es excluirla del modelo de ciudadanía hecho por y para los hombres. A pesar de todo esto, el término "ciudadana" se utiliza continuamente sobre todo por parte de las patriotas: les da la idea de participar en la vida política, a pesar de que signifique ser tan sólo un ciudadano pasivo (Sewell, 1988: 105-123).

Sin embargo, las autoras de La causa delle donne y La schiavitù delle donne trataron el tema con decisión y firmeza. La autora veneciana lo aborda en el segundo capítulo titulado "Le donne han diritto di essere a parte di tutti i pubblici interessi della presente riforma d'Italia" y que comprende los puntos 10-13 de su declaración. A lo largo del punto 10 va enumerando "i nostri diritti innegabili", empezando por el de participar en la esfera pública:

\section{noi abbiamo un vero diritto di essere a parte di tutti gli affari pubblici dipendenti dallo spirito e dall'intelletto, e questo nostro diritto è naturale, ed intrinsecamente annesso alla natura umana; diritto non inferiore al vostro, anzi tanto maggiore e più forte, quanto noi per lo spirito siamo più sublimi di voi. Abbiamo dunque diritto di assistere a tutte le vostre adunanze ed assemblee; abbiamo diritto di concorrere alla formazione delle leggi, alle quali dobbiamo del pari assoggettarci. Abbiamo diritto di sedere nei tribunali, nei magistrati, nei direttori esecutivi, abbiamo diritto di andare ai consolati, alle commissioni, alle ambasciate; abbiamo diritto di maneggiar le finanze, di governar le provincie, di regolare gli eserciti; abbiamo diritto di approvare o rip govare tutti i trattati nazionali o dic di alleanza o di guerra o di pace (La causa delle donne, 1964: 462).}

La mujer tiene que entrar en la esfera pública y así lo ha demostrado. Pero la ciudadana $^{* * *}$ siente también la necesidad de prever la reacción de muchos de sus compañeros, sobre todo el hecho de que muchos consideran que "gli affari domestici, propri del sesso feminino, c'impediscono l'esercizio di questi nostri diritti" (La causa delle donne, 1964: 462). Es, de nuevo, el tema de la relegación de la mujer a la esfera familiar el que va a tener que rebatir con fuerza, ya que, en última instancia, se trata del elemento crucial en la construcción de la identidad femenina en el discurso patriótico. 
Para nuestra autora tales ocupaciones son genéricas, no son propias de la mujer, incluso la educación de los hijos en los primeros años. Por tanto, se trata sólo de prejuicio el asignárselas exclusivamente a ellas:

Come potrà la vera sapienza comportare che tanti uomini stolti ed ignoranti, non capaci che della rocca e del fuso, sieno chiamati a consiglio per affari pubblici della maggior importanza; e tante donne all'incontro dotte, d'acuto ingegno, di posata prudenza abbiano da tener nascosti i loro sublimi talenti, dai qual grandissimo vantaggio ridonderebbe all'umanità! È ora che si tolga dal mondo questo perniciosissimo pregiudizio, come ne furono tolti tanti altri (La causa delle donne, 1964: 463)

Por otro lado, a los hombres el hecho de tener otras obligaciones nos les ha apartado nunca de la esfera pública: deberes privados y públicos son perfectamente compatibles. Por último, algunas mujeres ni siquiera tienen tales ocupaciones; por lo tanto, al menos a ellas se les deberían reconocer estos derechos políticos: "le fanciulle e le vedove formano un popolo innumerabile, e più inumerabile ancora vi riuscirà se vi porrete a canto tute le maritate, che o separate sono da' loro mariti o disgustate di loro". Por eso, se pregunta: "Perché tante donne, che formano la maggior parte del nostro sesso, dovranno essere private de' loro propri diritti naturali?" (La causa delle donne, 1964 463).

Los últimos dos capítulos de su discurso son recapitulativos: dadas todas estas premisas,

le nostre pretensioni sono giuste e fondate sulla legge naturale; dunque l'esclusione che foste per dare in appresso alle donne in tutti i vostri concessi, sarebbe un'esclusione contraria all'equità. Dunque tutti i progetti che da qui innanz si maneggieranno, tutte le leggi che si publicheranno, saranno invalide senza il nostro concorso. Dunque è vostro dovere il chiamar le donne a Consiglio per dare al sistema di libertà ed eguaglianza il conveniente vigore ed autenticità (La causa delle donne, 1964: 463-4).

Si no se tienen en cuenta sus reclamaciones, se estarán contradiciendo todos los ideales por los que se ha luchado hasta ahora:

Voi siete gli amanti della libertà, e non potrete soffrire che rimanga schiava una metà intiera del genere umano. Voi siete i difensori dell'eguaglianza, e non potrete far a meno di sostenere la causa di chi è simile a voi ed eguale vostro. Voi siete politici, e dovete conoscere per necessità che se il nostro sesso vi è amico, l'esecuzione del e dov vostro progetto è sicura; se è contrario a' vostri disegni, questi stesione del disegni saranno vani (La causa delle donne, 1964: 464)

Los demás patriotas lo deberían ver de ese modo, pero, añade la autora, si, a pesar de todo, deciden seguir como hasta ahora "siccome insieme con noi distruggereste tutti i nimici dell'uguaglianza, senza di noi non li distruggerete giammai" (La causa delle donne, 1964: 464).

Con estas palabras termina uno de los discursos más explícitos y valientes del Trienio jacobino italiano.

\section{REFERENCIAS BIBLIOGRÁFICAS}

Angelini, G. (a cura di), De amicitia: scritti dedicati a Arturo Colombo, Milano, Università Bocconi,

Aubert du Bayet, J.-B. A., Rapporto d'una festa civica celebrata in Costantinpoli da' Francesi e Veneziani riuniti per la felice rigenerazione di Venezia, Venezia, Zatta, 1797.

Barbiera, R., Il Salotto Della Contessa Maffei e la Società Milanese (1834-1886). London, 1895.

Bustico, G., "Il salotto milanese di un'Aspasia veneziana del periodo napoleonico", Nuovo Archivio Veneto, 33 (1917), pp. 370-78

Buttafuoco, A., "La causa delle donne. Cittadinanza e genere nel triennio 'giacobino' italiano", Modi di essere. Studi, riflessioni, interventi sulla cultura e la politica delle donne in onore di Elvira Badaracco, Bologna, EM Ricerche, 1991, pp. 99-106.

Buttafuoco, A., "Libertà, fraternità, uguaglianza: per chi? Donne nella rivoluzione francese", Esperienza storica femminile dell'età moderna e contemoranea, parte prima, Roma, Unione donne italiane, Circolo La Goccia, 1988, pp. 29-56.

Cicogna, E. A., Saggio di Bibliografia veneziana, Venezia, Tip. di G.B. Merlo, Venezia, 1847.

Cosmacini, G., Il medico giacobino. La vita e i tempi di Giovanni Rasori, Roma-Bari, Laterza, 2002.

Discorso della cittadina Annetta Vadori pronunciato nella Società di Pubblica Istruzione in occasione che fu invitata a pronunciare il giuramento solenne: vivere libera o morire, Venezia, Giovann Zatta, 1797.

Dumas, G., La fin de la République de Venise. Aspects et réflets littéraires, Paris, P.U.F., 1964.

Filippini, N. M. (a cura di), Donne sulla scena pubblica: società e politica in Veneto tra Sette e Ottocento, Roma, Franco Angeli, 2006

Filippini, N.M., "Annetta Vadori, rivoluzionaria vagabonda”, L'altra metà del Risorgimento. Volt e voci di patriote venete, a cura di Nadia Maria Filippi e Liviana Gazzetta, Verona, Cierre Edizioni, 2011, pp.114-116

I.P.M., Pensieri della libera cittadina IPM alle sue concittadine, Venezia, 1797.

Istruzioni d'una libera cittadina alle sue concittadine, Venezia, Andrea Martini, 1797.

"La causa delle donne. Discorso agl'italiani della cittadina*** (1797)", Cantimori, D.- De Felice, D. (a cura di), Giacobini italiani, Bari, Gius. Laterza \& Figli, 1964, vol.II, pp. 455-464. 
Lattanzi, C., La schiavitù delle donne. Memoria della cittadina L. letta all'Accademia di Pubblica Istruzione di Mantova, 14 Mietitore, Anno I della Libertà d'Italia. All'A pollo (1797), riedito a cura di Gilberto Zacchè, Mantova, Edizioni Lombarde, 1976.

Mattei, F., Dell'educazione che si deve dare alle donne, Vicenza, Bartolomeo Paroni,1797

Negri, T., “Discorso della Cittadina Teresa Negri Rasinelli pronunciato all’Unione del Pranzo Patriotico il dì 9 Partile anno 6. Repubblicano (28 Maggio 1798 v.s.)", en Marcelli, U. (a cura di), Il Gran Circolo Costituzionale e il "Genio Democratico" (Bologna 1797-98), Bologna, Analisi, 1986, vol.II, pp. 1095-99.

Pillinini, G., Venezia giacobina, Venezia, Editoria Universitaria, 1997.

Pisano, L- Veauvy, C., Parole inascoltate. Le donne e la costruzione dello Stato-nazione in Italia e in Francia (1789-1860), Roma, Editori Riuniti, 1994.

Plant, M., Venice: Fragile City, 1797-1997, Yale University Press, Yale, 2002.

Rao, A., Esuli. L'emigrazione italiana in Francia (1792-1802), Napoli, Guida, 1992.

Romanelli, G., Dai Dogi agli Imperatori: La fine della Repubblica tra storia e mito, Milano, Electa, 1997.

Stella, C., "Discorso pronunciato dalla cittadina Claudia Stella presidente della deputazione del pranzo patriotico delle cittadine bolognesi. Celebrato il dì 9. Pratile Anno 6 Repub. (28. Maggio 1798. v.s.)", en Marcelli, U. (a cura di), Il Gran Circolo Costituzionale e il "Genio Democratico" (Bologna 1797-98), Bologna, Analisi, 1986, vol.III, pp. 1103-1105.

Sewell, W. H., "Le citoyen/la citoyenne: activity, passivity, and the revolutionary concept of citizenship", C. Lucas (ed.), The French revolution and the creation of modern political culture, vol.2, The political culture of the French Revolution, Oxford-Toronto, Pergamon press, 1988, pp. 105-23. 